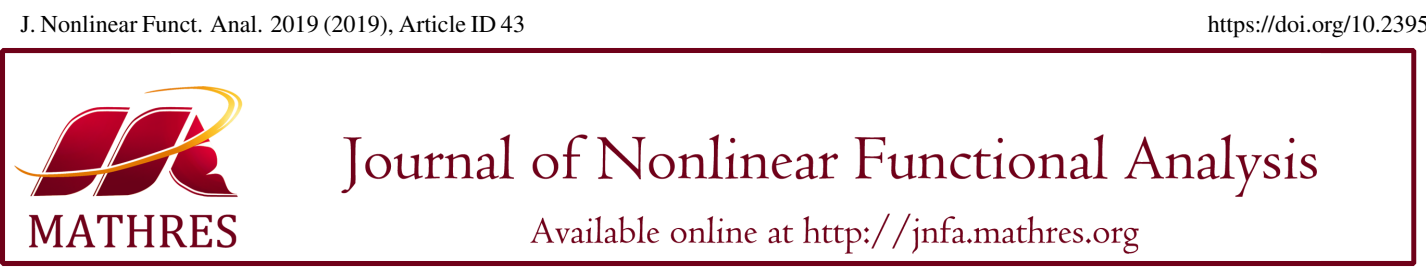

\title{
THE SPLIT EQUALITY FIXED-POINT PROBLEM FOR TOTALLY QUASI-ASYMPTOTICALLY STRICTLY PSEUDOCONTRACTIVE MAPPINGS WITHOUT PRIOR KNOWLEDGE OF OPERATOR NORMS
}

\author{
WEI LIU ${ }^{1}$, YAQIN WANG ${ }^{2, *}$, SUN YOUNG CHO ${ }^{3}$ \\ ${ }^{1}$ Department of Mathematics, Shaoxing University, Yuanpei College, Shaoxing 312000, China \\ ${ }^{2}$ Department of Mathematics, Shaoxing University, Shaoxing 312000, China \\ ${ }^{3}$ Department of Liberal Arts, Gyeongnam National University of Science and Technology, Gyeongnam, Korea
}

\begin{abstract}
The purpose of this paper is to consider the split equality fixed-point problem for totally quasi-asymptotically strictly pseudocontractive mappings without prior knowledge of operator norms in infinite-dimensional Hilbert spaces. Weak and strong convergence theorems are established. The results presented in this paper improve and extend some recent results. Keywords. Split equality fixed-point problem; Demiclosed; Semicompact; Weak and strong convergence.
\end{abstract}

2010 Mathematics Subject Classification. 47H09, 47N10, 47J05.

\section{INTRODUCTION}

Let $C$ and $Q$ be nonempty closed convex subsets of real Hilbert spaces $H_{1}$ and $H_{2}$, respectively, and let $A: H_{1} \rightarrow H_{2}$ be a bounded linear operator. The split feasibility problem (SFP) is to find a point $x^{*}$ satisfying

$$
x^{*} \in C, A x^{*} \in Q .
$$

The SFP (1.1) was first introduced by Censor and Elfving [1]. It serves as a model for many inverse problems where constraints are imposed on the solutions in the domain of a linear operator as well as in the range of the operator. There are a number of significant applications of the SFP in intensitymodulated radiation therapy, signal processing, image reconstruction and so on, and many well-known iterative algorithms for solving it were established; see, e.g., [2, 3, 4, 5, 6].

Recently, Moudafi [7] introduced a new split feasibility problem. Let $H_{1}, H_{2}, H_{3}$ be three Hilbert spaces, let $A: H_{1} \rightarrow H_{3}, B: H_{2} \rightarrow H_{3}$ be two bounded linear operators, and let $U: H_{1} \rightarrow H_{1}$ and $T:$

${ }^{*}$ Corresponding author.

E-mail address: wangyaqin0579@126.com (Y. Wang).

Received March 20, 2019; Accepted September 23, 2019.

(C)2019 Journal of Nonlinear Functional Analysis 
$H_{2} \rightarrow H_{2}$ be two firmly quasi-nonexpansive operators. The split equality fixed-point problem (SEFP) in [7] is to find

$$
x \in \operatorname{Fix}(U), y \in \operatorname{Fix}(T) \text { such that } A x=B y,
$$

which allows asymmetric and partial relations between the variables $x$ and $y$. The interest is to cover many situations, for instance, in decomposition methods for PDE's, applications in game theory and in intensity-modulated radiation therapy (IMRT). In decision sciences, this allows to consider agents who interplay only via some components of their decision variables (see [8]). In IMRT, this amounts to envisage a weak coupling between the vector of doses absorbed in all voxels and that of the radiation intensity (see [9]). Denote the solution set of SEFP by

$$
\Gamma=\left\{(x, y) \in H_{1} \times H_{2} \mid x \in F i x(U), y \in F i x(T), A x=B y\right\} .
$$

If $H_{2}=H_{3}$ and $B=I$, then problem (1.2) reduces to the split common fixed-point problem (SCFP):

$$
\text { find } x^{*} \in F i x(U) \text { such that } A x^{*} \in F i x(T) \text {, }
$$

which is a generalization of the SFP and the convex feasibility problem (CFP). The SCFP has been studied by many authors; see, e.g., [10, 11, 12, 13] and references therein.

For solving the SEFP, Moudifi and Al-Shemas [14] introduced the following simultaneous iterative algorithm:

$$
\left\{\begin{array}{l}
x_{k+1}=U\left(x_{k}-\gamma_{k} A^{*}\left(A x_{k}-B y_{k}\right)\right) \\
y_{k+1}=T\left(y_{k}+\gamma_{k} B^{*}\left(A x_{k}-B y_{k}\right)\right)
\end{array}\right.
$$

for firmly quasi-nonexpansive operators $U$ and $T$, where non-decreasing sequence $\gamma_{k} \in\left(\varepsilon, \frac{2}{\lambda_{A}+\lambda_{B}}-\varepsilon\right)$, and $\lambda_{A}, \lambda_{B}$ stand for the spectral radiuses of $A^{*} A$ and $B^{*} B$, respectively.

Note that in algorithm (1.3), the determination of the stepsize $\left\{\gamma_{k}\right\}$ depends on the operator norms $\|A\|$ and $\|B\|$ (or the largest eigenvalues of $A^{*} A$ and $B^{*} B$ ). For algorithm (1.3), one has first to compute (or estimate) operator norms of $A$ and $B$, which is in general not an easy work in practice. To overcome this difficulty, Lopez et al. [15] and Zhao and Yang [16] presented a helpful method for estimating the stepsizes which don't need prior knowledge of the operator norms for solving the split feasibility problems and multiple-set split feasibility problems, respectively. Inspired by them, Zhao [17] introduced the following algorithm:

$$
\left\{\begin{array}{l}
u_{k}=x_{k}-\gamma_{k} A^{*}\left(A x_{k}-B y_{k}\right), \\
x_{k+1}=\left(1-\alpha_{k}\right) u_{k}+\alpha_{k} U\left(u_{k}\right), \\
v_{k}=y_{k}+\gamma_{k} B^{*}\left(A x_{k}-B y_{k}\right), \\
y_{k+1}=\left(1-\beta_{k}\right) v_{k}+\beta_{k} T\left(v_{k}\right),
\end{array}\right.
$$

where the stepsize $\gamma_{k}$ is chosen in such a way that

$$
\gamma_{k} \in\left(0, \frac{2\left\|A x_{k}-B y_{k}\right\|^{2}}{\left\|A^{*}\left(A x_{k}-B y_{k}\right)\right\|^{2}+\left\|B^{*}\left(A x_{k}-B y_{k}\right)\right\|^{2}}\right) .
$$

The advantage of the stepsizes satisfying (1.5) lies in the fact that no prior information about the operators norms of $A$ and $B$. Under some suitable conditions, she proved the weak convergence of this algorithm to solve the SEFP (1.2) governed by quasi-nonexpansive operators. 
Very recently, Zhao and He [18] introduced the following iterative algorithm for solving the SEFP for generalized asymptotically quasi-nonexpansive mappings. Let $x_{0} \in H_{1}, y_{0} \in H_{2}$ be arbitrary, and the sequence $\left\{\left(x_{k}, y_{k}\right)\right\}$ be defined by

$$
\left\{\begin{array}{l}
u_{k}=x_{k}-\gamma_{k} A^{*}\left(A x_{k}-B y_{k}\right) \\
x_{k+1}=\left(1-\alpha_{k}\right) u_{k}+\alpha_{k} U^{k}\left(u_{k}\right), \\
v_{k}=y_{k}+\gamma_{k} B^{*}\left(A x_{k}-B y_{k}\right) \\
y_{k+1}=\left(1-\alpha_{k}\right) v_{k}+\alpha_{k} T^{k}\left(v_{k}\right),
\end{array}\right.
$$

where $\left\{\gamma_{k}\right\}$ is chosen by (1.5). They obtained the weak convergence of this algorithm under some suitable conditions in Hilbert spaces.

In this paper, motivated and inspired by the works mentioned above, we consider iterative algorithm (1.6) for solving the SEFP for totally quasi-asymptotically strictly pseudocontractive mappings which include generalized asymptotically quasi-nonexpansive mappings and asymptotically quasi-nonexpansive mappings without prior knowledge of operator norms. And we establish some weak convergence results under some suitable conditions in Hilbert spaces. Meanwhile, we note that the existing algorithms for the SEFP in $[17,18]$ have only weak convergence in the framework of infinite-dimensional spaces. And it is of interest to seek strong convergence for algorithm (1.6). Then we establish some strong convergence theorems for the SEFP.

\section{PRELIMINARIES}

Let $C$ be a nonempty closed convex subset of a real Hilbert space $H$ with inner product $\langle\cdot, \cdot\rangle$, and norm $\|\cdot\|$. When $\left\{x_{n}\right\}$ is a sequence in $H$, we denote the strong convergence of $\left\{x_{n}\right\}$ to $x \in H$ by $x_{n} \rightarrow x$ and the weak convergence by $x_{n} \rightarrow x$. Denote by Fix $(T)$ the set of fixed points of a mapping $T$; that is, $\operatorname{Fix}(T)=\{x \in C: T x=x\}$.

In order to facilitate our investigation in this paper, we recall some definitions as follows.

Definition 2.1. A mapping $T: C \rightarrow C$ is said to be

(i) nonexpansive if

$$
\|T x-T y\| \leq\|x-y\|, \forall x, y \in C ;
$$

(ii) quasi-nonexpansive if $\operatorname{Fix}(T) \neq \emptyset$ and

$$
\|T x-z\| \leq\|x-z\|, \quad \forall x \in C, z \in F i x(T) ;
$$

(iii) $\mu$-demicontractive (see for example [11]) if there exists a constant $\mu \in[0,1)$ such that

$$
\|T x-z\|^{2} \leq\|x-z\|^{2}+\mu\|x-T x\|^{2}, \forall(x, z) \in C \times F i x(T) .
$$

Definition 2.2. ([19, 20]). A mapping $T: C \rightarrow C$ is said to be

(i) $\left(\beta,\left\{\mu_{k}\right\},\left\{\xi_{k}\right\}, \phi\right)$-totally asymptotically strictly pseudocontractive, if there exist a constant $\beta \in[0,1)$, sequences $\left\{\mu_{k}\right\} \subset[0, \infty)$ and $\left\{\xi_{k}\right\} \subset[0, \infty)$ with $\mu_{k} \rightarrow 0$ and $\xi_{k} \rightarrow 0$ such that, for all $x, y \in C$,

$$
\left\|T^{k} x-T^{k} y\right\|^{2} \leq\|x-y\|^{2}+\beta\left\|x-y-\left(T^{k} x-T^{k} y\right)\right\|^{2}+\mu_{k} \phi(\|x-y\|)+\xi_{k}, k \geq 1,
$$

where $\phi:[0, \infty) \rightarrow[0, \infty)$ is a continuous and strictly increasing function with $\phi(0)=0$;

(ia) Especially, if $\phi(t)=t^{2}$ and $\xi_{k}=0$, then $T$ is said to be $\left(\beta,\left\{\mu_{k}\right\}\right)$-asymptotically strictly pseudocontractive; 
(ib) Especially, if $\phi(t)=t^{2}$ and $\beta=0$, then $T$ is said to be a generalized asymptotically nonexpansive mapping with $\left(\left\{\mu_{k}\right\},\left\{\xi_{k}\right\}\right)$;

(ic) Especially, if $\mu_{k}=\xi_{k}=0$, then $T$ is said to be $\beta$-strictly pseudocontractive;

(ii) $\left(\beta,\left\{\mu_{k}\right\},\left\{\xi_{k}\right\}, \phi\right)$-totally quasi-asymptotically strictly pseudocontractive, if $\operatorname{Fix}(T) \neq \emptyset$, and there exist a constant $\beta \in[0,1)$, sequences $\left\{\mu_{k}\right\} \subset[0, \infty)$ and $\left\{\xi_{k}\right\} \subset[0, \infty)$ with $\mu_{k} \rightarrow 0$ and $\xi_{k} \rightarrow 0$ such that for all $x \in C$

$$
\left\|T^{k} x-z\right\|^{2} \leq\|x-z\|^{2}+\beta\left\|x-T^{k} x\right\|^{2}+\mu_{k} \phi(\|x-z\|)+\xi_{k}, k \geq 1, \forall z \in \operatorname{Fix}(T),
$$

where $\phi:[0, \infty) \rightarrow[0, \infty)$ is a continuous and strictly increasing function with $\phi(0)=0$;

(iia) Especially, if $\phi(t)=t^{2}$ and $\xi_{k}=0$, then $T$ is said to be $\left(\beta,\left\{\mu_{k}\right\}\right)$-quasi-asymptotically strictly pseudocontractive;

(iib) Especially, if $\phi(t)=t^{2}$ and $\beta=0$, then $T$ is said to be a generalized asymptotically quasi-nonexpansive mapping with $\left(\left\{\mu_{k}\right\},\left\{\xi_{k}\right\}\right)$;

(iii) uniformly $L$-Lipschitzian if there exists a constant $L>0$ such that for all $x, y \in C$

$$
\left\|T^{k} x-T^{k} y\right\| \leq L\|x-y\|, \quad \forall k \geq 1 .
$$

Remark 2.3. If $T$ is a totally asymptotically strictly pseudocontractive mapping and $\operatorname{Fix}(T) \neq \emptyset$, then $T$ is a totally quasi-asymptotically strictly pseudocontractive mapping.

Definition 2.4. A mapping $T: C \rightarrow C$ is said to be

(i) demiclosed at the origin if, for any sequence $\left\{x_{k}\right\} \in H$ and $x^{*} \in H$, we have

$$
\left.\begin{array}{l}
x_{k} \rightarrow x^{*} \\
T\left(x_{k}\right) \rightarrow 0
\end{array}\right\} \Rightarrow T x^{*}=0 .
$$

(ii) semicompact if, for any bounded sequence $\left\{x_{k}\right\} \subset H$ with $\lim _{k \rightarrow \infty}\left\|x_{k}-T x_{k}\right\|=0$, there exists a subsequence $\left\{x_{k_{i}}\right\}$ of $\left\{x_{k}\right\}$ such that $\left\{x_{k_{i}}\right\}$ converges strongly to a point $x^{*} \in H$.

In a Hilbert space, we easily get the following equality:

$$
2\langle x, y\rangle=\|x\|^{2}+\|y\|^{2}-\|x-y\|^{2} .
$$

In what follows, we give some lemmas needed for the convergence analysis of our algorithms.

Lemma 2.5. [21] Let $H$ be a real Hilbert space. Then, for all $t \in[0,1]$ and $x, y \in H$,

$$
\|t x+(1-t) y\|^{2}=t\|x\|^{2}+(1-t)\|y\|^{2}-t(1-t)\|x-y\|^{2} .
$$

Lemma 2.6. Let $T: H \rightarrow H$ be $\left(\beta,\left\{\mu_{k}\right\},\left\{\xi_{k}\right\}, \phi\right)$-totally quasi-asymptotically strictly pseudocontractive, and set $T_{\alpha}^{k}=(1-\alpha) I+\alpha T^{k}$ for $\alpha \in(0,1]$. Then, for any $z \in F i x(T)$ and $x \in H$, one has the following:

$$
\left\|T_{\alpha}^{k} x-z\right\|^{2} \leq\|x-z\|^{2}+\alpha \mu_{k} \phi(\|x-z\|)+\alpha \xi_{k}-\alpha(1-\alpha-\beta)\left\|T^{k} x-x\right\|^{2} .
$$

Proof. It follows from Lemma 2.5 that

$$
\begin{aligned}
\left\|T_{\alpha}^{k} x-z\right\|^{2}= & \left\|(1-\alpha)(x-z)+\alpha\left(T^{k} x-z\right)\right\|^{2} \\
= & (1-\alpha)\|x-z\|^{2}+\alpha\left\|T^{k} x-z\right\|^{2}-\alpha(1-\alpha)\left\|T^{k} x-x\right\|^{2} \\
\leq & (1-\alpha)\|x-z\|^{2}-\alpha(1-\alpha)\left\|T^{k} x-x\right\|^{2} \\
& +\alpha\left[\|x-z\|^{2}+\beta\left\|x-T^{k} x\right\|^{2}+\mu_{k} \phi(\|x-z\|)+\xi_{k}\right] \\
= & \|x-z\|^{2}+\alpha \mu_{k} \phi(\|x-z\|)+\alpha \xi_{k}-\alpha(1-\alpha-\beta)\left\|T^{k} x-x\right\|^{2} .
\end{aligned}
$$


Lemma 2.7. [22]. Suppose $\left\{a_{k}\right\}$ is a sequence of nonnegative real numbers such that

$$
a_{k+1} \leq\left(1+\delta_{k}\right) a_{k}+b_{k}, k \geq 0 .
$$

If (i) $\sum_{k=0}^{\infty} \delta_{k}<\infty$ and (ii) $\sum_{k=0}^{\infty} b_{k}<\infty$, then $\lim _{k \rightarrow \infty} a_{k}$ exists. In particular, if $\left\{a_{k}\right\}$ has a subsequence which converges strongly to zero, then $\lim _{k \rightarrow \infty} a_{k}=0$.

Proposition 2.8. [19] Let $H$ be a real Hilbert space and let $T: H \rightarrow H$ be a uniformly L-Lipschitzian and $\left.\left(\gamma,\left\{\mu_{n}\right\},\left\{\xi_{n}\right\}, \phi\right\}\right)$-totally asymptotically strictly pseudocontractive mapping. Then the demiclosedness principle holds for $T$ in the sense that if $\left\{x_{n}\right\}$ is a sequence in $H$ such that $x_{n} \rightarrow x^{*}$, and

$$
\limsup _{m \rightarrow \infty} \limsup _{n \rightarrow \infty}\left\|x_{n}-T^{m} x_{n}\right\|=0,
$$

then $(I-T) x^{*}=0$. In particular, if $x_{n} \rightarrow x^{*}$, and $\left\|(I-T) x_{n}\right\| \rightarrow 0$, then $(I-T) x^{*}=0$, that is, $I-T$ is demiclosed at the origin.

\section{MAIN RESULTS}

In this section we will prove the weak and strong convergence of the algorithm (1.6) to solve the SEFP (1.2).

Now let us assume that the following conditions are satisfied.

(H1) $H_{1}, H_{2}, H_{3}$ are three Hilbert spaces, $A: H_{1} \rightarrow H_{3}, B: H_{2} \rightarrow H_{3}$ are two bounded linear operators.

(H2) $U: H_{1} \rightarrow H_{1}$ is a uniformly $L_{1}$-Lipschitzian and $\left(\beta_{1},\left\{\mu_{k}^{(1)}\right\},\left\{\xi_{k}^{(1)}\right\}, \phi_{1}\right)$-totally quasi-asymptotically strictly pseudocontractive mapping and $T: H_{2} \rightarrow H_{2}$ is a uniformly $L_{2}$-Lipschitzian and $\left(\beta_{2},\left\{\mu_{k}^{(2)}\right\},\left\{\xi_{k}^{(2)}\right\}, \phi_{2}\right)$ totally quasi-asymptotically strictly pseudocontractive mapping satisfying the following conditions:

(C1) $\mu_{k}=\max \left\{\mu_{k}^{(1)}, \mu_{k}^{(2)}\right\}, \xi_{k}=\max \left\{\xi_{k}^{(1)}, \xi_{k}^{(2)}\right\}, k \geq 0$ and $\Sigma_{k=0}^{\infty} \mu_{k}<\infty, \Sigma_{k=0}^{\infty} \xi_{k}<\infty$.

(C2) $\phi=\max \left\{\phi_{1}, \phi_{2}\right\}$ and there exist two positive constants $M$ and $M^{*}$ such that $\phi(\lambda) \leq M^{*} \lambda^{2}$ for all $\lambda \geq M$.

(H3) The stepsize $\gamma_{k}$ in the algorithm (1.6) is chosen in such a way that

$$
\gamma_{k} \in\left(0, \frac{2\left\|A x_{k}-B y_{k}\right\|^{2}}{\left\|A^{*}\left(A x_{k}-B y_{k}\right)\right\|^{2}+\left\|B^{*}\left(A x_{k}-B y_{k}\right)\right\|^{2}}\right), k \in \Omega,
$$

otherwise, $\gamma_{k}=\gamma$ ( $\gamma$ being any nonnegative value), where the set of indexes $\Omega=\left\{k: A x_{k}-B y_{k} \neq 0\right\}$.

Lemma 3.1. [17] Assume the solution set $\Gamma$ of the SEFP (1.2) is nonempty. Then, $\gamma_{k}$ defined by (3.1) is well defined.

Theorem 3.2. Let $H_{1}, H_{2}, H_{3}, A, B, U, T,\left\{\mu_{k}\right\},\left\{\xi_{k}\right\}$ and $\phi$ satisfy the assumptions (H1)-(H3) mentioned before. Let $x_{0} \in H_{1}, y_{0} \in H_{2}$ be arbitrary, the sequence $\left\{\left(x_{k}, y_{k}\right)\right\}$ be defined by (1.6). Assume, for small enough $\varepsilon>0$,

$$
\gamma_{k} \in\left(\varepsilon, \frac{2\left\|A x_{k}-B y_{k}\right\|^{2}}{\left\|A^{*}\left(A x_{k}-B y_{k}\right)\right\|^{2}+\left\|B^{*}\left(A x_{k}-B y_{k}\right)\right\|^{2}}-\varepsilon\right),
$$

where $k \in \Omega$, otherwise, $\gamma_{k}=\gamma\left(\gamma\right.$ being any nonnegative value). Let $\left\{\alpha_{k}\right\} \subset(\delta, 1-\delta-\beta)$ for a small enough $\delta>0$, where $\beta=\max \left\{\beta_{1}, \beta_{2}\right\}$. Assume that $I-T$ and $I-U$ are demiclosed at the origin. (I) If $\Gamma \neq \emptyset$, then $\left\{\left(x_{k}, y_{k}\right)\right\}$ weakly converges to a solution $\left(x^{*}, y^{*}\right)$ of the $\operatorname{SEFP}(1.2)$. 
(II) In addition, if $U$ and $T$ are also semicompact, then $\left\{\left(x_{k}, y_{k}\right)\right\}$ strongly converges to a solution $\left(x^{*}, y^{*}\right)$ of the SEFP (1.2).

Proof. (I) Repeating the proof of Theorem 2.4 in [17], we know that $\left\{\gamma_{k}\right\}$ is bounded. For any $(x, y) \in \Gamma$, i.e., $x \in F i x(U), y \in F i x(T)$ and $A x=B y$, by (1.6) and (2.1), we have

$$
\begin{aligned}
\left\|u_{k}-x\right\|^{2}= & \left\|x_{k}-x-\gamma_{k} A^{*}\left(A x_{k}-B y_{k}\right)\right\|^{2} \\
= & \left\|x_{k}-x\right\|^{2}-2 \gamma_{k}\left\langle A^{*}\left(A x_{k}-B y_{k}\right), x_{k}-x\right\rangle+\gamma_{k}^{2}\left\|A^{*}\left(A x_{k}-B y_{k}\right)\right\|^{2} \\
= & \left\|x_{k}-x\right\|^{2}-2 \gamma_{k}\left\langle A x_{k}-B y_{k}, A x_{k}-A x\right\rangle+\gamma_{k}^{2}\left\|A^{*}\left(A x_{k}-B y_{k}\right)\right\|^{2} \\
= & \left\|x_{k}-x\right\|^{2}-\gamma_{k}\left[\left\|A x_{k}-B y_{k}\right\|^{2}+\left\|A x_{k}-A x\right\|^{2}-\left\|B y_{k}-A x\right\|^{2}\right] \\
& +\gamma_{k}^{2}\left\|A^{*}\left(A x_{k}-B y_{k}\right)\right\|^{2} .
\end{aligned}
$$

Similarly, by (1.6) and (2.1), we have

$$
\begin{aligned}
\left\|v_{k}-y\right\|^{2}= & \left\|y_{k}-y\right\|^{2}-\gamma_{k}\left[\left\|A x_{k}-B y_{k}\right\|^{2}+\left\|B y_{k}-B y\right\|^{2}-\left\|A x_{k}-B y\right\|^{2}\right] \\
& +\gamma_{k}^{2}\left\|B^{*}\left(A x_{k}-B y_{k}\right)\right\|^{2} .
\end{aligned}
$$

Combining (3.3), (3.4) and $A x=B y$, we have

$$
\begin{aligned}
\left\|u_{k}-x\right\|^{2}+\left\|v_{k}-y\right\|^{2} \leq & \left\|x_{k}-x\right\|^{2}+\left\|y_{k}-y\right\|^{2}-\gamma_{k}\left[2\left\|A x_{k}-B y_{k}\right\|^{2}\right. \\
& \left.-\gamma_{k}\left(\left\|A^{*}\left(A x_{k}-B y_{k}\right)\right\|^{2}+\left\|B^{*}\left(A x_{k}-B y_{k}\right)\right\|^{2}\right)\right] .
\end{aligned}
$$

Since $\phi$ is a continuous and increasing function, it results that $\phi(\lambda) \leq \phi(M)$, if $\lambda \leq M$, and $\phi(\lambda) \leq M^{*} \lambda^{2}$, if $\lambda \geq M$. In either case, we can obtain that

$$
\phi(\lambda) \leq \phi(M)+M^{*} \lambda^{2}, \forall \lambda \geq 0 .
$$

By (1.6), (3.6) and Lemma 2.6, we obtain

$$
\begin{aligned}
\left\|x_{k+1}-x\right\|^{2} \leq & \left\|u_{k}-x\right\|^{2}+\alpha_{k} \mu_{k} \phi\left(\left\|u_{k}-x\right\|\right) \\
& +\alpha_{k} \xi_{k}-\alpha_{k}\left(1-\alpha_{k}-\beta_{1}\right)\left\|U^{k}\left(u_{k}\right)-u_{k}\right\|^{2} \\
\leq & \left\|u_{k}-x\right\|^{2}+\alpha_{k} \mu_{k}\left(\phi(M)+M^{*}\left\|u_{k}-x\right\|^{2}\right) \\
& +\alpha_{k} \xi_{k}-\alpha_{k}\left(1-\alpha_{k}-\beta_{1}\right)\left\|U^{k}\left(u_{k}\right)-u_{k}\right\|^{2} \\
= & \left(1+\alpha_{k} \mu_{k} M^{*}\right)\left\|u_{k}-x\right\|^{2}+\alpha_{k} \mu_{k} \phi(M) \\
& +\alpha_{k} \xi_{k}-\alpha_{k}\left(1-\alpha_{k}-\beta_{1}\right)\left\|U^{k}\left(u_{k}\right)-u_{k}\right\|^{2}
\end{aligned}
$$

and

$$
\begin{aligned}
\left\|y_{k+1}-y\right\|^{2} \leq & \left\|v_{k}-y\right\|^{2}+\alpha_{k} \mu_{k} \phi\left(\left\|v_{k}-y\right\|\right) \\
& +\alpha_{k} \xi_{k}-\alpha_{k}\left(1-\alpha_{k}-\beta_{2}\right)\left\|T^{k}\left(v_{k}\right)-v_{k}\right\|^{2} \\
\leq & \left\|v_{k}-y\right\|^{2}+\alpha_{k} \mu_{k}\left(\phi(M)+M^{*}\left\|v_{k}-y\right\|^{2}\right) \\
& +\alpha_{k} \xi_{k}-\alpha_{k}\left(1-\alpha_{k}-\beta_{2}\right)\left\|T^{k}\left(v_{k}\right)-v_{k}\right\|^{2} \\
= & \left(1+\alpha_{k} \mu_{k} M^{*}\right)\left\|v_{k}-y\right\|^{2}+\alpha_{k} \mu_{k} \phi(M) \\
& +\alpha_{k} \xi_{k}-\alpha_{k}\left(1-\alpha_{k}-\beta_{2}\right)\left\|T^{k}\left(v_{k}\right)-v_{k}\right\|^{2} .
\end{aligned}
$$


By the above two inequalities and (3.5), we obtain

$$
\begin{aligned}
& \left\|x_{k+1}-x\right\|^{2}+\left\|y_{k+1}-y\right\|^{2} \\
\leq & \left(1+\alpha_{k} \mu_{k} M^{*}\right)\left(\left\|u_{k}-x\right\|^{2}+\left\|v_{k}-y\right\|^{2}\right)+2 \alpha_{k} \mu_{k} \phi(M)+2 \alpha_{k} \xi_{k} \\
& -\alpha_{k}\left(1-\alpha_{k}-\beta_{1}\right)\left\|U^{k}\left(u_{k}\right)-u_{k}\right\|^{2}-\alpha_{k}\left(1-\alpha_{k}-\beta_{2}\right)\left\|T^{k}\left(v_{k}\right)-v_{k}\right\|^{2} \\
\leq & \left(1+\alpha_{k} \mu_{k} M^{*}\right)\left(\left\|x_{k}-x\right\|^{2}+\left\|y_{k}-y\right\|^{2}\right)-\left(1+\alpha_{k} \mu_{k} M^{*}\right) \gamma_{k}\left[2\left\|A x_{k}-B y_{k}\right\|^{2}\right. \\
& \left.-\gamma_{k}\left(\left\|A^{*}\left(A x_{k}-B y_{k}\right)\right\|^{2}+\left\|B^{*}\left(A x_{k}-B y_{k}\right)\right\|^{2}\right)\right]+2 \alpha_{k} \mu_{k} \phi(M)+2 \alpha_{k} \xi_{k} \\
& -\alpha_{k}\left(1-\alpha_{k}-\beta\right)\left\|U^{k}\left(u_{k}\right)-u_{k}\right\|^{2}-\alpha_{k}\left(1-\alpha_{k}-\beta\right)\left\|T^{k}\left(v_{k}\right)-v_{k}\right\|^{2} .
\end{aligned}
$$

Setting $\rho_{k}(x, y)=\left\|x_{k}-x\right\|^{2}+\left\|y_{k}-y\right\|^{2}$, we have

$$
\begin{aligned}
& \rho_{k+1}(x, y) \\
\leq & \left(1+\alpha_{k} \mu_{k} M^{*}\right) \rho_{k}(x, y)-\left(1+\alpha_{k} \mu_{k} M^{*}\right) \gamma_{k}\left[2\left\|A x_{k}-B y_{k}\right\|^{2}\right. \\
& \left.-\gamma_{k}\left(\left\|A^{*}\left(A x_{k}-B y_{k}\right)\right\|^{2}+\left\|B^{*}\left(A x_{k}-B y_{k}\right)\right\|^{2}\right)\right]+2 \alpha_{k} \mu_{k} \phi(M)+2 \alpha_{k} \xi_{k} \\
& -\alpha_{k}\left(1-\alpha_{k}-\beta\right)\left\|U^{k}\left(u_{k}\right)-u_{k}\right\|^{2}-\alpha_{k}\left(1-\alpha_{k}-\beta\right)\left\|T^{k}\left(v_{k}\right)-v_{k}\right\|^{2},
\end{aligned}
$$

which, together with (3.2), implies that

$$
\rho_{k+1}(x, y) \leq\left(1+\alpha_{k} \mu_{k} M^{*}\right) \rho_{k}(x, y)+2 \alpha_{k} \mu_{k} \phi(M)+2 \alpha_{k} \xi_{k} .
$$

Consequently, taking $\delta_{k}=\alpha_{k} \mu_{k} M^{*}, b_{k}=2 \alpha_{k} \mu_{k} \phi(M)+2 \alpha_{k} \xi_{k}$, we have from Lemma 2.7 and (C1) that $\left\{\rho_{k}(x, y)\right\}$ converges to some finite limit, denoted by $\rho(x, y)$. Therefore noting that $A x_{k}-B y_{k}=0$, if $k \notin \Omega$, it follows from (3.2), (3.7) and the conditions on $\left\{\alpha_{k}\right\},\left\{\mu_{k}\right\}$, and $\left\{\xi_{k}\right\}$ that

$$
\begin{aligned}
& \lim _{k \rightarrow \infty}\left[2\left\|A x_{k}-B y_{k}\right\|^{2}-\gamma_{k}\left(\left\|A^{*}\left(A x_{k}-B y_{k}\right)\right\|^{2}+\left\|B^{*}\left(A x_{k}-B y_{k}\right)\right\|^{2}\right)\right]=0, \\
& \lim _{k \rightarrow \infty}\left\|U^{k}\left(u_{k}\right)-u_{k}\right\|=0, \\
& \lim _{k \rightarrow \infty}\left\|T^{k}\left(v_{k}\right)-v_{k}\right\|=0 .
\end{aligned}
$$

Furthermore, by (3.2) and (3.8), we have

$$
\lim _{k \rightarrow \infty}\left(\left\|A^{*}\left(A x_{k}-B y_{k}\right)\right\|^{2}+\left\|B^{*}\left(A x_{k}-B y_{k}\right)\right\|^{2}\right)=0 .
$$

So it follows from (3.8) and (3.10) that

$$
\lim _{k \rightarrow \infty}\left\|A x_{k}-B y_{k}\right\|=0
$$

And (3.10) implies that

$$
\lim _{k \rightarrow \infty}\left\|A^{*}\left(A x_{k}-B y_{k}\right)\right\|=\lim _{k \rightarrow \infty}\left\|B^{*}\left(A x_{k}-B y_{k}\right)\right\|=0 .
$$

Furthermore, since $\left\{\gamma_{k}\right\}$ is bounded, we obtain

$$
\lim _{k \rightarrow \infty}\left\|u_{k}-x_{k}\right\|=\lim _{k \rightarrow \infty} \gamma_{k}\left\|A^{*}\left(A x_{k}-B y_{k}\right)\right\|=0 .
$$

It follows from (1.6) that

$$
\begin{aligned}
\left\|x_{k+1}-x_{k}\right\| & \leq\left(1-\alpha_{k}\right)\left\|u_{k}-x_{k}\right\|+\alpha_{k}\left\|U^{k}\left(u_{k}\right)-x_{k}\right\| \\
& \leq\left(1-\alpha_{k}\right)\left\|u_{k}-x_{k}\right\|+\alpha_{k}\left\|U^{k}\left(u_{k}\right)-u_{k}\right\|+\alpha_{k}\left\|u_{k}-x_{k}\right\| \\
& =\left\|u_{k}-x_{k}\right\|+\alpha_{k}\left\|U^{k}\left(u_{k}\right)-u_{k}\right\|,
\end{aligned}
$$


which, together with (3.9) and (3.12), implies that

$$
\lim _{k \rightarrow \infty}\left\|x_{k+1}-x_{k}\right\|=0
$$

Similarly, we have

$$
\begin{aligned}
\lim _{k \rightarrow \infty}\left\|v_{k}-y_{k}\right\| & =0, \\
\lim _{k \rightarrow \infty}\left\|y_{k+1}-y_{k}\right\| & =0 .
\end{aligned}
$$

It follows from (3.12) and (3.13) that

$$
\left\|u_{k+1}-u_{k}\right\| \leq\left\|u_{k+1}-x_{k+1}\right\|+\left\|x_{k+1}-x_{k}\right\|+\left\|x_{k}-u_{k}\right\| \rightarrow 0(k \rightarrow \infty) .
$$

Next we show that $\lim _{k \rightarrow \infty}\left\|U\left(u_{k}\right)-u_{k}\right\|=0$ and $\lim _{k \rightarrow \infty}\left\|T\left(v_{k}\right)-v_{k}\right\|=0$. Set $c_{k}=\left\|U^{k}\left(u_{k}\right)-u_{k}\right\|$, Since $U$ is uniformly $L_{1}$-Lipschitzian, we get

$$
\begin{aligned}
\left\|U\left(u_{k}\right)-u_{k}\right\| \leq & \left\|U^{k}\left(u_{k}\right)-u_{k}\right\|+\left\|U^{k}\left(u_{k}\right)-U\left(u_{k}\right)\right\| \\
\leq & c_{k}+L_{1}\left\|U^{k-1}\left(u_{k}\right)-u_{k}\right\| \\
\leq & c_{k}+L_{1}\left(\left\|U^{k-1}\left(u_{k}\right)-U^{k-1}\left(u_{k-1}\right)\right\|\right. \\
& \left.+\left\|U^{k-1}\left(u_{k-1}\right)-u_{k-1}\right\|+\left\|u_{k}-u_{k-1}\right\|\right) \\
\leq & c_{k}+L_{1}\left(L_{1}\left\|u_{k}-u_{k-1}\right\|+c_{k-1}\right)+L_{1}\left(\left\|u_{k}-u_{k-1}\right\|\right) .
\end{aligned}
$$

Therefore, we obtain from (3.9) and (3.14) that

$$
\lim _{k \rightarrow \infty}\left\|U\left(u_{k}\right)-u_{k}\right\|=0
$$

Similarly, we have

$$
\lim _{k \rightarrow \infty}\left\|T\left(v_{k}\right)-v_{k}\right\|=0 .
$$

Taking $\left(x^{*}, y^{*}\right) \in \omega_{w}\left(x_{k}, y_{k}\right)$, we have $x^{*} \in \omega_{w}\left(x_{k}\right)$ and $y^{*} \in \omega_{w}\left(y_{k}\right) . \lim _{k \rightarrow \infty}\left\|u_{k}-x_{k}\right\|=0$ and $\lim _{k \rightarrow \infty} \| v_{k}-$ $y_{k} \|=0$ imply that $x^{*} \in \omega_{w}\left(u_{k}\right)$ and $y^{*} \in \omega_{w}\left(v_{k}\right)$. Since $I-T$ and $I-U$ are demiclosed at the origin, we have from (3.15) and (3.16) that $x^{*} \in F i x(U)$ and $y^{*} \in F i x(T)$. Also $A x^{*}-B y^{*} \in \omega_{w}\left(A x_{k}-B y_{k}\right)$, which together with the weakly lower semicontinuity of the norm and (3.11) implies that

$$
\left\|A x^{*}-B y^{*}\right\| \leq \liminf _{k \rightarrow \infty}\left\|A x_{k}-B y_{k}\right\|=0 .
$$

Hence $\left(x^{*}, y^{*}\right) \in \Gamma$. Following the last part of the proof in Theorem 2.4 in [17], we have the uniqueness of the weak cluster points of $\left\{x_{k}\right\}$ and $\left\{y_{k}\right\}$. Then $\left\{\left(x_{k}, y_{k}\right)\right\}$ generated by (1.6) weakly converges to a solution $\left(x^{*}, y^{*}\right)$ of the $\operatorname{SEFP}(1.2)$.

(II) It follows from $\lim _{k \rightarrow \infty} \rho_{k}(x, y)=\rho(x, y)$ that $\left\{x_{k}\right\}$ and $\left\{y_{k}\right\}$ are bounded. Then by $\lim _{k \rightarrow \infty} \| u_{k}-$ $x_{k} \|=0$ and $\lim _{k \rightarrow \infty}\left\|v_{k}-y_{k}\right\|=0$, we get that $\left\{u_{k}\right\}$ and $\left\{v_{k}\right\}$ are also bounded. Since $U$ is semi-compact, it follows from (3.15) that there exists a subsequence $\left\{u_{k_{j}}\right\} \subset\left\{u_{k}\right\}$ such that $u_{k_{j}} \rightarrow u^{*} \in H_{1} . x_{k_{j}} \rightarrow x^{*}$ and $\lim _{k \rightarrow \infty}\left\|A^{*}\left(A x_{k}-B y_{k}\right)\right\|=0$ imply that

$$
u_{k_{j}}=x_{k_{j}}-\gamma_{k_{j}} A^{*}\left(A x_{k_{j}}-B y_{k_{j}}\right) \rightarrow x^{*}(j \rightarrow \infty) .
$$


So $u^{*}=x^{*}$, i.e., $u_{k_{j}} \rightarrow x^{*}$. Thus by (3.12) we have $x_{k_{j}} \rightarrow x^{*}$. Similarly, we can prove that $y_{k_{j}} \rightarrow y^{*}$ (Necessary we may take the subsequence of $\left.\left\{k_{j}\right\}\right)$. Since $\lim _{k \rightarrow \infty} \rho_{k}\left(x^{*}, y^{*}\right)=\rho\left(x^{*}, y^{*}\right)$, where $\rho_{k}\left(x^{*}, y^{*}\right)=$ $\left\|x_{k}-x^{*}\right\|^{2}+\left\|y_{k}-y^{*}\right\|^{2}$, we have $\lim _{j \rightarrow \infty} \rho_{k_{j}}\left(x^{*}, y^{*}\right)=0$. So it follows from Lemma 2.7 that

$$
\lim _{k \rightarrow \infty} \rho_{k}\left(x^{*}, y^{*}\right)=0
$$

which implies that $\lim _{k \rightarrow \infty}\left\|x_{k}-x^{*}\right\|=0$ and $\lim _{k \rightarrow \infty}\left\|y_{k}-y^{*}\right\|=0$, i.e., $\left\{\left(x_{k}, y_{k}\right)\right\}$ generated by (1.6) strongly converges to a solution $\left(x^{*}, y^{*}\right)$ of the SEFP (1.2). This competes the proof.

Theorem 3.3. Let $H_{1}, H_{2}, H_{3}, A, B,\left\{\mu_{k}\right\},\left\{\xi_{k}\right\}$ and $\phi$ be the same as Theorem 3.2. Let $U: H_{1} \rightarrow H_{1}$ be a uniformly $L_{1}$-Lipschitzian and $\left(\beta_{1},\left\{\mu_{k}^{(1)}\right\},\left\{\xi_{k}^{(1)}\right\}, \phi_{1}\right)$-totally asymptotically strictly pseudocontractive mapping and $\mathrm{T}: \mathrm{H}_{2} \rightarrow \mathrm{H}_{2}$ be a uniformly $L_{2}$-Lipschitzian and $\left(\beta_{2},\left\{\mu_{k}^{(2)}\right\},\left\{\xi_{k}^{(2)}\right\}, \phi_{2}\right)$-totally asymptotically strictly pseudocontractive mapping. Let $x_{0} \in H_{1}, y_{0} \in H_{2}$ be arbitrary, the sequence $\left\{\left(x_{k}, y_{k}\right)\right\}$ be defined by (1.6). Assume for small enough $\varepsilon>0$,

$$
\gamma_{k} \in\left(\varepsilon, \frac{2\left\|A x_{k}-B y_{k}\right\|^{2}}{\left\|A^{*}\left(A x_{k}-B y_{k}\right)\right\|^{2}+\left\|B^{*}\left(A x_{k}-B y_{k}\right)\right\|^{2}}-\varepsilon\right),
$$

where $k \in \Omega$, otherwise, $\gamma_{k}=\gamma\left(\gamma\right.$ being any nonnegative value). Let $\left\{\alpha_{k}\right\} \subset(\delta, 1-\delta-\beta)$ for a small enough $\delta>0$, where $\beta=\max \left\{\beta_{1}, \beta_{2}\right\}$.

(I) If $\Gamma \neq \emptyset$, then $\left\{\left(x_{k}, y_{k}\right)\right\}$ weakly converges to a solution $\left(x^{*}, y^{*}\right)$ of the $\operatorname{SEFP}(1.2)$.

(II) In addition, if $U$ and $T$ are also semicompact, then $\left\{\left(x_{k}, y_{k}\right)\right\}$ strongly converges to a solution $\left(x^{*}, y^{*}\right)$ of the SEFP (1.2).

Proof. By Proposition 2.8, we have that $I-T$ and $I-U$ are demiclosed at the origin. And $\Gamma \neq \emptyset$ implies that $\operatorname{Fix}(U) \neq \emptyset$ and $F i x(T) \neq \emptyset$. Using Theorem 3.2 and Remark 2.3, we obtain the conclusions.

Remark 3.4. By Theorem 3.3, both $U$ and $T$ are reduced to $\left(\beta,\left\{\mu_{k}\right\}\right)$-asymptotically strictly pseudocontractive mappings or generalized asymptotically nonexpansive mappings with $\left(\left\{\mu_{k}\right\},\left\{\xi_{k}\right\}\right)$, and we can obtain the corresponding weak and strong convergence for the sequence $\left\{\left(x_{k}, y_{k}\right)\right\}$ defined by (1.6).

The following theorems can be concluded from Theorem 3.2 immediately.

Theorem 3.5. Let $H_{1}, H_{2}, H_{3}, A, B,\left\{\mu_{k}\right\}$ be the same as Theorem 3.2. Let $U: H_{1} \rightarrow H_{1}$ be a uniformly $L_{1}$ Lipschitzian and $\left(\beta_{1},\left\{\mu_{k}^{(1)}\right\}\right)$-quasi-asymptotically strictly pseudocontractive mapping and $\mathrm{T}: \mathrm{H}_{2} \rightarrow \mathrm{H}_{2}$ be a uniformly $L_{2}$-Lipschitzian and $\left(\beta_{2},\left\{\mu_{k}^{(2)}\right\}\right)$-quasi-asymptotically strictly pseudocontractive mapping. Let $x_{0} \in H_{1}, y_{0} \in H_{2}$ be arbitrary, the sequence $\left\{\left(x_{k}, y_{k}\right)\right\}$ be defined by (1.6). Assume for small enough $\varepsilon>0$,

$$
\gamma_{k} \in\left(\varepsilon, \frac{2\left\|A x_{k}-B y_{k}\right\|^{2}}{\left\|A^{*}\left(A x_{k}-B y_{k}\right)\right\|^{2}+\left\|B^{*}\left(A x_{k}-B y_{k}\right)\right\|^{2}}-\varepsilon\right),
$$

where $k \in \Omega$, otherwise, $\gamma_{k}=\gamma\left(\gamma\right.$ being any nonnegative value). Let $\left\{\alpha_{k}\right\} \subset(\delta, 1-\delta-\beta)$ for a small enough $\delta>0$, where $\beta=\max \left\{\beta_{1}, \beta_{2}\right\}$. Assume that $I-T$ and $I-U$ are demiclosed at the origin.

(I) If $\Gamma \neq \emptyset$, then $\left\{\left(x_{k}, y_{k}\right)\right\}$ weakly converges to a solution $\left(x^{*}, y^{*}\right)$ of the $\operatorname{SEFP}(1.2)$.

(II) In addition, if $U$ and $T$ are also semicompact, then $\left\{\left(x_{k}, y_{k}\right)\right\}$ strongly converges to a solution $\left(x^{*}, y^{*}\right)$ of the SEFP (1.2). 
Theorem 3.6. Let $H_{1}, H_{2}, H_{3}, A, B,\left\{\mu_{k}\right\},\left\{\xi_{k}\right\}$ be the same as Theorem 3.2. Let $U: H_{1} \rightarrow H_{1}$ be a uniformly $L_{1}$-Lipschitzian and generalized asymptotically quasi-nonexpansive mapping with $\left(\left\{\mu_{k}^{(1)}\right\},\left\{\xi_{k}^{(1)}\right\}\right)$ and $\mathrm{T}: \mathrm{H}_{2} \rightarrow \mathrm{H}_{2}$ be a uniformly $\mathrm{L}_{2}$-Lipschitzian and generalized asymptotically quasi-nonexpansive mapping with $\left(\left\{\mu_{k}^{(2)}\right\},\left\{\xi_{k}^{(2)}\right\}\right)$. Let $x_{0} \in H_{1}, y_{0} \in H_{2}$ be arbitrary, the sequence $\left\{\left(x_{k}, y_{k}\right)\right\}$ be defined by (1.6). Assume for small enough $\varepsilon>0$,

$$
\gamma_{k} \in\left(\varepsilon, \frac{2\left\|A x_{k}-B y_{k}\right\|^{2}}{\left\|A^{*}\left(A x_{k}-B y_{k}\right)\right\|^{2}+\left\|B^{*}\left(A x_{k}-B y_{k}\right)\right\|^{2}}-\varepsilon\right),
$$

where $k \in \Omega$, otherwise, $\gamma_{k}=\gamma\left(\gamma\right.$ being any nonnegative value). Let $\left\{\alpha_{k}\right\} \subset(\delta, 1-\delta)$ for a small enough $\delta>0$. Assume that $I-T$ and $I-U$ are demiclosed at the origin.

(I) If $\Gamma \neq \emptyset$, then $\left\{\left(x_{k}, y_{k}\right)\right\}$ weakly converges to a solution $\left(x^{*}, y^{*}\right)$ of the SEFP (1.2).

(II) In addition, if $U$ and $T$ are also semicompact, then $\left\{\left(x_{k}, y_{k}\right)\right\}$ strongly converges to a solution $\left(x^{*}, y^{*}\right)$ of the SEFP (1.2).

Remark 3.7. Since $\mu_{k} \geq 0, \xi_{k} \geq 0, \forall k \geq 0$, it is easy to see that $\sum_{k=0}^{\infty} \mu_{k}<\infty, \sum_{k=0}^{\infty} \xi_{k}<\infty \Leftrightarrow \sum_{k=0}^{\infty}\left(\mu_{k}+\right.$ $\left.\xi_{k}\right)<\infty$. Thus Theorem 3.6 (I) is Theorem 2.4 in [18]. Moreover under the conditions of Theorem 3.6 we also obtain the strong convergence for the sequence $\left\{\left(x_{k}, y_{k}\right)\right\}$ defined by (1.6).

Remark 3.8. Theorem 3.2 improves and extends the corresponding results of Zhao and He [18], Chang et al. [19] and so on.

Example 3.9. [23] Let $H=R$. Let $T: R \rightarrow R$ be defined by

$$
T x=\left\{\begin{array}{l}
a x, \quad \text { if } 0 \leq x \leq 1 / 2, \\
\frac{a}{2 a-1}(a-x), \text { if } 1 / 2 \leq x \leq a, \\
0, \text { if } x<0 \text { or } x>a,
\end{array}\right.
$$

where $1 / 2<a<1$. Then $\operatorname{Fix}(T)=\{0\}, T$ is a uniformly Lipschitzian, totally quasi-asymptotically strictly pseudocontractive mapping. Meanwhile, $I-T$ is demiclosed at 0 , but $T$ is not nonexpansive.

Proof. Clearly, $F(T)=\{0\}$. Since $1 / 2<a<1$, if $1 / 2 \leq x \leq a$, then $0 \leq \frac{a}{2 a-1}(a-x) \leq a / 2(\leq 1 / 2)$. Now we show that $T$ is a uniformly Lipschitzian, totally quasi-asymptotically strictly pseudocontractive mapping and $I-T$ is demiclosed at the origin.

Indeed, if $0 \leq x \leq 1 / 2$ and $1 / 2 \leq y \leq a$, then $T^{k} x=a^{k} x$ and $T^{k} y=\frac{a^{k}}{2 a-1}(a-y)$. We observe

$$
\begin{aligned}
\left|T^{k} x-T^{k} y\right| & =\left|a^{k} x-\frac{a^{k}}{2}+\frac{a^{k}}{2}-\frac{a^{k}}{2 a-1}(a-y)\right| \\
& =\left|a^{k}\left(x-\frac{1}{2}\right)+\frac{a^{k}}{2 a-1}\left[\left(a-\frac{1}{2}\right)-(a-y)\right]\right| \\
& \leq a^{k}\left|x-\frac{1}{2}\right|+\frac{a^{k}}{2 a-1}\left|y-\frac{1}{2}\right| \\
& \leq \frac{a^{k}}{2 a-1}|x-y| \\
& \leq l_{k}|x-y| \\
& =|x-y|+\left(l_{k}-1\right)|x-y|,
\end{aligned}
$$

where $l_{k}=1+\frac{a^{k}}{2 a-1}$. Let $L=\sup _{k \geq 1}\left\{l_{k}\right\}$. Since $\frac{a^{k}}{2 a-1} \rightarrow 0, T$ is $\left.\left(0,\left\{l_{k}-1\right\},\left\{\xi_{k}\right\}, \phi\right\}\right)$-totally asymptotically strictly pseudocontractive mapping, where $\phi(t)=t^{2}$. By Lemma 2.8, $I-T$ is demiclosed at the 
origin. Since $\operatorname{Fix}(T) \neq \emptyset, T$ is $\left.\left(0,\left\{l_{k}-1\right\},\left\{\xi_{k}\right\}, \phi\right\}\right)$-totally quasi-asymptotically strictly pseudocontractive mapping, moreover, $T$ is uniformly $L$-Lipschitzian. However, if taking $1 / 2 \leq x, y \leq a$, by noticing $\frac{a}{2 a-1}>1, \frac{1}{2 a-1}>1 \Leftrightarrow a<1$, we have

$$
|T x-T y|=\frac{a}{2 a-1}|x-y|>|x-y|,
$$

which implies that $T$ is not nonexpansive.

Next, we give a remark satisfying the conditions in Theorem 3.2.

Remark 3.10. Let $H_{1}=H_{2}=H_{3}=R$. Take $U=T$ as Example 3.9. We define a bounded linear operator $A: R \rightarrow R$ by $A x=3 x$. Thus $A^{*} x=3 x$ and $\|A\|=\left\|A^{*}\right\|=3$. Let $B=A$. Since $\operatorname{Fix}(U)=F i x(T)=\{0\}$, $\Gamma=\{(0,0)\} \neq \emptyset$. For small enough $\varepsilon>0$, since

$$
\begin{aligned}
& \frac{2\left\|A x_{k}-B y_{k}\right\|^{2}}{\left\|A^{*}\left(A x_{k}-B y_{k}\right)\right\|^{2}+\left\|B^{*}\left(A x_{k}-B y_{k}\right)\right\|^{2}} \\
\geq & \frac{2\left\|A x_{k}-B y_{k}\right\|^{2}}{\left\|A^{*}\right\|^{2}\left\|A x_{k}-B y_{k}\right\|^{2}+\left\|B^{*}\right\|^{2}\left\|A x_{k}-B y_{k}\right\|^{2}} \\
= & \frac{2}{\left\|A^{*}\right\|^{2}+\left\|B^{*}\right\|^{2}} \\
= & 1 / 9,
\end{aligned}
$$

we just need to take $\varepsilon \in(0,1 / 18)$. Then for each $k \geq 0$, choose $\left\{\gamma_{k}\right\}$ satisfying the condition (3.2). From the definitions of $U$ and $T, \beta_{1}=\beta_{2}=0$, so for any small enough $\delta>0$, we can choose $\left\{\alpha_{k}\right\} \subset(\delta, 1-\delta)$.

\section{Funding}

The paper was supported by Research Program on the Reform of Teaching Methodology in Higher Education, Zhejiang Province (no. JG20160261), the Natural Science Foundation of China (no. 11401388, 11671365, 11975156) and Gyeongnam National University of Science and Technology, Korea (Grant in 2018.3-2020.2).

\section{REFERENCES}

[1] Y. Censor, T. Elfving, A multiprojection algorithm using Bregman projections in a product space, Numer. Algo. 8 (1994), 221-239.

[2] H.K. Xu, Iterative methods for the split feasibility problem in infinite-dimensional Hilbert spaces, Inverse Probl. 26 (2010), Article ID 105018.

[3] X. Qin, L. Wang, A fixed point method for solving a split feasibility problem in Hilbert spaces, RACSAM Rev. R. Acad. Cienc. Exactas Fis. Nat. Ser. A Mat. 113 (2019), 315-325.

[4] Y.Y. Huang, C.C. Hong, Approximating common fixed points of averaged self-mappings with applications to the split feasibility problem and maximal monotone operators in Hilbert spaces, Fixed Point Theory Appl. 2013 (2013), Article ID 190.

[5] F.H. Wang, H.K. Xu, Approximating curve and strong convergence of the CQ algorithm for the split feasibility problem, J. Inequal. Appl. 2010 (2010), Article ID 102085.

[6] X. Qin, A. Petrusel, J.C. Yao, CQ iterative algorithms for fixed points of nonexpansive mappings and split feasibility problems in Hilbert spaces, J. Nonlinear Convex Anal. 19 (2018), 157-165.

[7] A. Moudafi, Alternating CQ-algorithm for convex feasibility and split fixed-point problems, J. Nonlinear Convex Anal. 15 (2014), 809-818. 
[8] H. Attouch, J. Bolte, P. Redont, A. Soubeyran, Alternating proximal algorithms for weakly coupled minimization problems. Applications to dynamical games and PDE's, J. Convex Anal. 15 (2008), 485-506.

[9] Y. Censor, T. Bortfeld, B. Martin, A. Trofimov, A unified approach for inversion problems in intensity-modulated radiation therapy, Phys. Med. Biol. 51 (2006), 2353-2365.

[10] Y. Censor, A. Segal, The split common fixed point problem for directed operators, J. Convex Anal. 16 (2009), 587-600.

[11] A. Moudafi, The split common fixed-point problem for demicontractive mappings, Inverse Probl. 26 (2010), 055007.

[12] F. Wang, H.K. Xu, Weak and strong convergence of two algorithms for the split fixed point problem, Numer. Math. Theor. Meth. Appl. 11 (2018), 770-781.

[13] X. Qin, J.C. Yao, A viscosity iterative method for a split feasibility problem, J. Nonlinear Convex Anal. 20 (2019), 14971506.

[14] A. Moudafi, E. Al-Shemas, Simultaneous iterative methods for split equality problem, Trans. Math. Program. Appl. 1 (2013), 1-11.

[15] G. Lopez, V. Martin-Marquez, F.H. Wang, H.K. Xu, Solving the split feasibility problem without prior knowledge of matrix norms, Inverse Probl. 28 (2012), Article ID 085004.

[16] J. Zhao, Q. Yang, A simple projection method for solving the multiple-sets split feasibility problem, Inverse Probl. Sci. Eng. 21 (2013), 537-546.

[17] J. Zhao, Solving split equality fixed-point problem of quasi-nonexpansive mappings without prior knowledge of operators norms, Optimization 64 (2015), 2619-2630.

[18] J. Zhao, S. He, Simultaneous iterative algorithms for the split common fixed-point problem of generalized asymptotically quasi-nonexpansive mappings without prior knowledge of operator norms, Fixed Point Theory Appl. 2014 (2014), Article ID 73.

[19] S.S. Chang, L. Wang, Y.K. Tang, L. Yang, The split common fixed point problem for total asymptotically strictly pseudocontractive mappings, J. Appl. Math. 2012 (2012), Artical ID 385638.

[20] P. Wang, H. Zhou, Strong convergence algorithms of the split common fixed point problem for total quasi-asymptotically pseudocontractive operators, Abstr. Appl. Anal. 2014 (2014), Artical ID 309368.

[21] C. Matinez-Yanes, H.K. Xu, Strong convergence of the CQ method for fixed point iteration processes, Nonlinear Anal. 64 (2006), 2400-2411.

[22] G. Crombez, A geometrical look at iterative methods for operators with fixed points, Numer. Func. Anal. Optim. 26 (2005), 157-175.

[23] T.H. Kim, D.H. Kim, Demiclosedness principle for continuous TAN mappings, ”Nonlinear Analysis and Convex Analysis” W. Takahashi and T. Tanaka, Eds., RIMS Kôykûroku 1821, 2013, 90-106. 